\title{
Aggravating influence of atomoxetine on the severity of stuttering and its successful treatment with methylphenidate: a case report
}

\author{
Ayla Uzun Cicek ${ }^{1 \oplus}$ \\ ${ }^{1}$ Cumhuriyet University, Faculty of Medicine, Department of Child and Adolescent Psychiatry, Sivas - Turkey
}

\begin{abstract}
Stuttering is a communication disorder characterized by a disruption in normal fluency and time patterning of speech. None of the pharmacological agents for stuttering could be recommended for general use; specific drug treatment that is simultaneously effective for both stuttering and attention deficit hyperactivity disorder (ADHD) is currently unknown. In this article, we present a case affected by severe developmental stuttering and ADHD that was exacerbated by atomoxetine but favorably responded to treatment with methylphenidate.
\end{abstract}

Keywords: Atomoxetine, attention deficit hyperactivity disorder, child/adolescent, methylphenidate, stuttering

\section{INTRODUCTION}

Stuttering is a communication disorder defined as a disruption in the normal fluency and time patterning of speech. Its main characteristics include frequent prolongations and/or repetitions of spoken sounds and/ or syllables, audible or silent blocking, interjections, and word production with excess physical tension $(1,2)$.

Stuttering has a lifetime prevalence of up to $8.5 \%$ and affects especially boys. The most common pattern, appearing during childhood, is developmental stuttering. Stuttering is often self-limiting and approximately $75 \%$ of cases resolve spontaneously. Speech therapy is the main treatment modality of stuttering (2).

A relationship between stuttering and attention skills is known. Recently, a study has reported that $58 \%$ of stutterers have symptoms of attention deficit hyperactivity disorder (ADHD) (3).

No pharmacological agent without unacceptable side effects has been recommended for general use. Accordingly, specific drug treatment that is simultaneously effective for both stuttering and ADHD is currently unknown. Yet, it has been recommended to avoid psychostimulants that increase dopamine and to opt for the use of atomoxetine or clonidine when the two disorders co-exist (4). On the other hand, several case reports and clinical anecdotes have reported both favorable (5-7) and adverse $(8,9)$ consequences regarding the effects of methylphenidate on stuttering.

No data has been found to link the use of the nonstimulant atomoxetine with the onset and/or exacerbation of stuttering. A beneficial effect of atomoxetine on stuttering has even been reported (10).

How to cite this article: Uzun Cicek A. Aggravating influence of atomoxetine on the severity of stuttering and its successful treatment with methylphenidate: a case report. Dusunen Adam The Journal of Psychiatry and Neurological Sciences 2020;33:210-213.

Correspondence: Ayla Uzun Cicek, Cumhuriyet University, Faculty of Medicine, Department of Child and Adolescent Psychiatry, Zip Code: 58140 Sivas - Turkey 
Here, we present the case of an adolescent affected by severe developmental stuttering and ADHD whose condition was exacerbated by atomoxetine but favorably responded to treatment with methylphenidate. Written informed consent for the presentation and publication of the case was obtained from the patient and his parents on condition that the patient's anonymity must be preserved.

\section{CASE}

A 14-year-old male was referred to the clinic with complaints of attention deficit, concentration difficulties, and stuttering since the age of 7 years. Primary complaints were attention problems, and poor organizational skills caused by these problems were an obstacle for the high school entrance exam. In addition, the patient's social functioning was impaired because of stuttering. He had never received speech therapy due to financial and transport limitations. The relationship of stuttering with anxiety and social situations was not immediately obvious. Severe stuttering and secondary symptoms in his efforts to get his words out were detected; severely disrupting his communication. He did not suffer from dysarthria or aphasia.

After clinical examination and receiving information from the family and the patient, the latter was diagnosed with developmental stuttering and ADHD predominantly inattentive presentation according to the DSM-5 (1). His neurology and otorhinolaryngology consultation, all routine laboratory investigations, thyroid function tests, and electrocardiogram showed no abnormality. His ADHD diagnosis had been made at age 7 , but he had not used the recommended medication. There were no important features in his background. All developmental steps, including speech-language development phases, had been timely. He did not have epileptic seizures, traumas, or other significant physical diseases. In his family history, his uncle had permanent developmental stuttering.

He was prescribed $25 \mathrm{mg} /$ day of atomoxetine gradually increased to $40 \mathrm{mg} /$ day. Unfortunately, stuttering was appreciably exacerbated within the third week of treatment, so the dose of atomoxetine was reduced back to $25 \mathrm{mg} /$ day. However, the worsening of stuttering did not regress. The patient and his family reported periods of variable length, lasting from 10 days to 3 months, during which the frequency and intensity of stuttering increased or decreased without any obvious triggering factor or event in the patient's history; this increase reached its peak with the use of atomoxetine. The expected effect of the atomoxetine for ADHD was also not realized. Thus atomoxetine was terminated in the $7^{\text {th }}$ week of treatment. Stuttering regressed to the previous level after 10 days of stopping the medication.

At this point, the patient was started on $10 \mathrm{mg} /$ day methylphenidate, which was increased to $18 \mathrm{mg} /$ day from the second week. At follow-up 3 weeks later, his stuttering was dramatically reduced and the severity of ADHD symptoms greatly diminished. Treatment was continued with methylphenidate at a fixed dose of 27 $\mathrm{mg} /$ day for more than one year with sustained improvement of the stuttering. Even though no measuring instrument was used to assess the stuttering severity during the follow-up period, the patient and his family stated that stuttering was significantly reduced, and this improvement was observed during the interviews as well.

\section{DISCUSSION}

This case describes an unfavorable reaction to atomoxetine, exacerbating stuttering in a patient who presented with comorbid stuttering and ADHD. However, he demonstrated a remarkable relief of the stuttering after medication with methylphenidate.

Two results are important in the treatment of the case presented here. Firstly, this may be the first presentation of a stuttering case that was exacerbated by atomoxetine, according to a review of the literature at the time of writing up this report. Conversely, there is evidence that the use of atomoxetine reduces symptoms not only of ADHD but also of stuttering behavior (10). Atomoxetine is a selective norepinephrine reuptake inhibitor; its mechanism of action is to increase the release of norepinephrine and dopamine in the prefrontal cortex. It may be recommended as a priority in patients with ADHD who have comorbid anxiety disorders or depression (11). Even if the relationship between our patient's stutter moments and anxiety is very weak, it is still interesting to note that stuttering is markedly aggravated with atomoxetine. This dramatic worsening may result from atomoxetine affecting the dopaminergic system indirectly by raising the noradrenaline level. Atomoxetine simultaneously also increases extracellular levels of dopamine as well as noradrenaline in the prefrontal cortex, because there are very few dopamine transporter proteins in the 
prefrontal cortex (11-13). Thus, one could speculate that atomoxetine might mediate stuttering by affecting dopamine levels.

Secondly, this case report is one of the few observations to illustrate a striking improvement of stuttering with methylphenidate. In the literature, there are conflicting results regarding the effects of methylphenidate on stuttering. Several case reports have suggested that methylphenidate clearly improves stuttering (5-7), while others have reported methylphenidate to have adverse effects $(8,9)$. The first clinical evidence for stuttering associated with the use of methylphenidate was presented by Alparslan et al. (9). Nevertheless, methylphenidate has not been extensively studied for stuttering treatment; therefore, it is difficult to reach a definite conclusion about the effect of methylphenidate on speech fluency, though one study has shown that methylphenidate provides a significant improvement in stuttering compared to placebo (6).

Our patient's pronounced positive response to methylphenidate can be explained by the effect of methylphenidate on the dopaminergic system, which is also implicated in the stuttering mechanism. Some stuttering adults are cured with dopamine 1 (D1) or dopamine 2 (D2) blockers, while others worsen (4). Methylphenidate stimulates D1 receptors and reduces D2 receptors by indirect stimulation (7). Moreover, striato-cortico-thalamic pathways, which have an important role in executive functions, are involved in both ADHD and stuttering. Methylphenidate enhances executive functions. Because verbal fluency is also considered to be one of the executive functions, methylphenidate may have affected stuttering through improving executive functions generally $(6,7)$. However, given the contradictory effects of methylphenidate on stuttering, there may be other mechanisms than these.

We have presented an interesting finding regarding the treatment of stuttering that initially deteriorated with atomoxetine and significantly improved with methylphenidate. Although no similar cases have been reported in the literature, clinicians should be aware that atomoxetine may have an adverse effect on the severity of stuttering. On the other hand, methylphenidate may be an option for treatment of stuttering in cases presenting with comorbid stuttering and ADHD. Further extensive studies with larger samples are needed to confirm or refute the contradictory findings obtained from this case. Future studies replicating research with these agents will improve our understanding of stuttering and the neurobiological basis of ADHD.

\begin{tabular}{|l|l|l|}
\hline \multicolumn{4}{|l|}{ Contribution Categories } & Author Initials \\
\hline \multirow{4}{*}{ Category 1} & Concept/Design & A.U.C. \\
\cline { 2 - 3 } & Literature review & A.U.C. \\
\cline { 2 - 3 } & Data analysis/Interpretation & A.U.C. \\
\cline { 2 - 3 } & Case follow-up (if applicable) & A.U.C. \\
\hline \multirow{3}{*}{ Category 2} & Drafting manuscript & A.U.C. \\
\cline { 2 - 3 } & Critical revision of manuscript & A.U.C. \\
\hline Category 3 & Final approval and accountability & A.U.C. \\
\hline \multirow{3}{*}{ Other } & Technical or material support & A.U.C. \\
\cline { 2 - 3 } & Supervision & A.U.C. \\
\hline
\end{tabular}

Acknowledgments: The author wants to thank the patient and his family for collaborating in the study.

Informed Consent: Written informed consent for case presentation and publication was obtained from patients and his parents, on condition that the patient's anonymity must be preserved.

Peer-review: Externally peer-reviewed.

Conflict of Interest: The authors reported no conflict of interest related to this article.

Financial Disclosure: There are no financial, personal, or professional interests.

\section{REFERENCES}

1. American Psychiatric Association. Diagnostic and Statistical Manual of Mental Disorders (DSM-5). Fifth ed., Washington, DC: American Psychiatric Association, 2013.

2. Sadock BJ, Sadock VA, Ruiz P. Kaplan and Sadock's synopsis of psychiatry, behavioral sciences/clinical psychiatry. Eleventh Ed. Philadelphia: Wolters Kluwer, 2015; 1147-1149.

3. Donaher J, Richels C. Traits of attention deficit/hyperactivity disorder in school-age children who stutter. J Fluency Disord 2012; 37:242-252.

4. Maguire GA, Yu BP, Franklin DL, Riley GD. Alleviating stuttering with pharmacological interventions. Expert Opin Pharmacother 2004; 5:1565-1571.

5. Devroey D, Beerens G, Van De Vijver E. Methylphenidate as a treatment for stuttering: a case report. Eur Rev Med Pharmacol Sci 2012; 16:66-69.

6. Rabaeys H, Bijleveld HA, Devroey D. Influence of methylphenidate on the frequency of stuttering: a randomized controlled trial. Ann Pharmacother 2015; 49:1096-1104.

7. Bodur S, Isildar Y, Kara H, Sabanci M. Effectiveness of osmoticcontrolled release oral delivery system methylphenidate in the treatment of stuttering that combined with attention-deficit/ hyperactivity disorder: a case report. Am J Psychiatry Neurosci 2014; 2:115-117.

8. Copur M, Copur S. Emergence of stuttering in an attention deficit hyperactivity disorder patient treated with methylphenidate. Dusunen Adam The Journal of Psychiatry and Neurological Sciences 2018; 31:222-224. 
9. Alpaslan AH, Coskun KŞ, Kocak U, Gorucu Y. Stuttering associated with the use of short-acting oral methylphenidate. J Clin Psychopharmacol 2015; 35:739-741.

10. Donaher J, Healey EC, Zobell A. The effects of ADHD medication changes on a child who stutters. Perspect Fluen Fluen Disord 2009; 19:95-98.

11. Zhou J. Norepinephrine transporter inhibitors and their therapeutic potential. Drugs Future 2004; 29:1235-1244.
12. Kratochvil CJ, Vaughan BS, Harrington MJ, Burke WJ. Atomoxetine: a selective noradrenaline reuptake inhibitor for the treatment of attention-deficit/hyperactivity disorder. Expert Opin Pharmacother 2003; 4:1165-1174.

13. Morón JA, Brockington A, Wise RA, Rocha BA, Hope BT. Dopamine uptake through the norepinephrine transporter in brain regions with low levels of the dopamine transporter: evidence from knock-out mouse lines. J Neurosci 2002; 22:389-395. 